\title{
Multilinguales
}

\section{Le discours paremiologique beti dans Le sorcier signe et persiste de Camille Nkoa Atenga}

Beti pariological discourse in Le sorcier signe et persiste of Camille Nkoa Atenga

\section{Mouhamadou Ngapout Kpoumié}

\section{(2) OpenEdition}

\section{Journals}

Édition électronique

URL : http://journals.openedition.org/multilinguales/3925

DOI : 10.4000 /multilinguales.3925

ISSN : 2335-1853

\section{Éditeur}

Université Abderrahmane Mira - Bejaia

\section{Référence électronique}

Mouhamadou Ngapout Kpoumié, « Le discours paremiologique beti dans Le sorcier signe et persiste de Camille Nkoa Atenga », Multilinguales [En ligne], 11 | 2019, mis en ligne le 17 juillet 2019, consulté le 02 juin 2020. URL : http://journals.openedition.org/multilinguales/3925 ; DOI : https://doi.org/10.4000/ multilinguales.3925

Ce document a été généré automatiquement le 2 juin 2020

\section{(c) (†) $\ominus$}

Multilinguales est mise à disposition selon les termes de la Licence Creative Commons Attribution Pas d'Utilisation Commerciale - Pas de Modification 4.0 International 


\title{
Le discours paremiologique beti dans Le sorcier signe et persiste de Camille Nkoa Atenga
}

\author{
Beti pariological discourse in Le sorcier signe et persiste of Camille Nkoa
}

Atenga

Mouhamadou Ngapout Kpoumié

1 Depuis quelques décennies, on assiste à un renouvellement esthétique dans la littérature africaine francophone en général et camerounaise en particulier. En effet, le contact avec l'altérité occidentale a influencé les modes de faire, de se vêtir, de penser et d'être des peuples africains au point où l'on assiste à la perte des valeurs africaines. En fait, si on part du postulat que chaque peuple, chaque communauté humaine fonctionne sur la base des mentalités incrustées dans les esprits de ses membres, cellesci guident et façonnent leurs valeurs, leurs croyances, leurs coutumes, leurs langues et leurs traditions. L'on ne saurait refléter mieux son histoire, son patrimoine, sa façon d'exprimer ses idées et créativités sans elles. Ces habitudes s'érigent en des lois et en des codes de conduite qui, très souvent, déterminent les actions, les réactions, les aspirations, la vision du monde et même l'imagination des personnes prises isolément ou bien collectivement dans leur univers social. Face à ce problème de déclin de la civilisation orale, l'écrivain africain resté au pays, notamment, se mue en patriarche, courroie de transmission et de sauvegarde des valeurs ancestrales. En effet, celui-ci fait partie d'une communauté sociale qui possède un code de conduite, un système de valeurs partagées par les membres et dont il se propose d'en assurer la transmission. Se développe alors l'établissement d'un nouveau code d'écriture qui passe par la prise en compte de son environnement socioculturel notamment la préservation de l'oralité africaine.

2 Plusieurs textes issus du corpus littéraire africain sont en effet marqués

du sceau de cette tradition que les auteurs veulent préserver. C'est le cas spécifique de Le Sorcier signe et persiste ${ }^{1}$ (2003) de Camille Nkoa Atenga, roman qui s'enracine fortement dans l'univers socioculturel beti à travers notamment son art oral 
traditionnel à savoir le chant du muet, la berceuse, le chant épique, les mythes et les légendes de la société beti, les parémies. Analysant le cas précis de ces dernières, cette réflexion postule que l'auteur camerounais, par son écriture, circonscrit son terroir et tente de sauvegarder les valeurs traditionnelles beti portées par elles. Pour valider cette hypothèse, nous emprunterons à l'ethnostylistique théorisée par Gervais Mendo Ze qui entend replacer « chaque objet culturel, notamment chaque texte, au sein de la culture où il a été élaboré » (Mendo Ze, 2017 : 7). Après une étude des ethnostylèmes qui inscrivent le terroir d'énonciation du texte, nous mènerons une étude structurale des parémies beti avant d'en dégager l'incidence identitaire.

\section{Le terroir beti : lieu-source d'énonciation}

Dans son ouvrage Ethnostylistique. Une approche néo-structurale, Mendo Ze pose comme postulat que tout texte est enraciné dans un terroir culturel précis. De ce fait, toute lecture herméneutique de celui-ci suppose la prise en compte des éléments extratextuels qui irradient et éclairent son processus de signification. Il écrit justement à propos: "La problématique de l'approche ethnostylistique stipule que le texte, en tant qu'énoncé, ne peut se comprendre sans prendre en compte les circonstances dans lesquelles l'acte d'énonciation a pris place » (Mendo Ze, 2017: 54). Dans cette perspective, étudier un texte c'est retrouver des indices ou encore des ethnostylèmes qui permettent la saisie du contexte d'énonciation. Au nombre de ces indices, le critique propose une typologie qu'il synthétise dans ces propos :

Les indices fonctionnent comme des marqueurs, des indicateurs de culturalité. Ce sont des ethnostylèmes constitués par des mots, des expressions, des groupes de mots, des noms des personnes, d'animaux, de lieux ou de choses, des constructions phrastiques entières ou partielles permettant de réaliser la recherche de l'identification des éléments d'enracinement du texte dans son contexte et en extension (Mendo Ze, 2017 : 108).

Dans Le Sorcier signe et persiste en effet, le lecteur note un ensemble d'éléments extratextuels qui l'informent sur le lieu-source d'énonciation. En effet, le parcours de l'intrigue de ce roman s'apparente à une excursion dans le terroir beti tant les indices géographiques et culturels sont expressifs. Intéressons-nous tout d'abord aux indices onomastiques.

\subsection{Les marqueurs onomastiques}

5 L'onomastique est l'étude des noms propres au sens large. Elle se subdivise en deux branches à savoir l'anthroponymie qui est l'étude des noms propres et la toponymie qui est l'étude des noms des lieux. Selon Mendo Ze, elle est un marqueur de territoire puisqu'elle informe sur l'environnement immédiat ou sur l'« hypoculture » (Mendo Ze, 2017 : 109) de l'auteur dont et d'où il ne peut se désolidariser.

\section{I.1.1. Les référents toponymiques}

6 À la lecture de Le Sorcier signe et persiste de Nkoa Atenga, le lecteur est en présence d'une abondance d'ethnostylèmes géographiques qui informent notamment sur « les lieux, les espaces, aux pays » (Mendo Ze, 2017 : p. 108) où se déroule l'intrigue. Par le « consensus homotopique ${ }^{2} "$, c'est-à-dire une correspondance exacte entre l'espace 
textualisé et le référent réel, on circonscrit aisément le lieu-source d'énonciation. En effet dès l'entame du roman, le narrateur présente cet espace : « Ngoulmekong, petit village de campagne à une cinquantaine de kilomètres à l'ouest de Yaoundé » (Le Sorcier : 9). Même si l'auteur considère cet espace d'abord comme imaginaire, il va par la suite donner des détails qui précisent clairement le lieu d'ancrage du récit. Jugeonsen dans les propos ci-après :

Ainsi en est-il de Ngoulmekong ${ }^{3}$. Littéralement, « force de lances ». Les lances, diton et soutient-on ici, qui, jointes à la force des ancêtres farouchement déterminés, ont permis d'arracher ces terres dans le sang et à d'autres tribus - les Bassas - et de maintenir dissuasivement ces derniers au-delà de la grande rivière Lobo qui fait désormais frontière naturelle. La Lobo qui, entre autres, reçoit les eaux de la Mbema. La Mbema qui serpente la forêt et dont la grande particularité est précisément de singulariser Ngoulmekong qu'elle subdivise en trois hameaux pour avoir, en demi-lune, traversé à deux endroits, la portion de piste au long et de part et d'autre de laquelle s'étire langoureusement le village Eton. Un village qui fait à la fois suite et complément à une multitude d'autres villages semblables. Des villages auxquels mènent tous les chemins. À deux conditions cependant, pour celui qui veut se rendre à l'un d'entre eux : en connaître à la fois le nom ainsi que celui de la tribu qui l'occupe. Car il se trouve, non seulement ici en pays Eton mais à travers toute l'étendue de l'immense région du Nyong et Sanaga [...] (Le Sorcier : 10).

7 Ce passage laisse voir un ensemble d'ethnostylèmes géographiques ou "indices absolus " (Mendo Ze, 2017 : 108) qui rendent compte de l'inscription territoriale de l'auteur. Bien plus, le romancier donne d'autres indices de référence absolue qui guident le lecteur. Il en est ainsi de "Nlong " (Le Sorcier : 41), localité située au sudouest de Yaoundé, Lobo (70), Okola (25), Mbalmayo (51), Victoria (111), à l'est et au nord Cameroun (53), etc. Ces référents géographiques constituent autant de réalèmes, d'ethnostylèmes géographiques qui ancrent le roman de Nkoa Atenga dans un territoire précis. On peut de ce fait affirmer sans ambages que l'aire culturelle en présence est le territoire beti en particulier et camerounais en général. Et le romancier de préciser en note de bas de page à propos: "Beti. Le plus grand regroupement ethnico-socio-culturel du Nyong et Sanaga » (Le Sorcier : 11).

\subsubsection{Les référents anthroponymiques}

8 Parce qu'empreints d'une forte charge culturelle, les désignateurs anthroponymiques trahissent l'inscription territoriale de l'auteur. De ce fait, ils véhiculent un capital symbolique et marquent l'imaginaire collectif des membres du groupe. Ainsi, on constate que le nom renvoie à un territoire, un groupe socioculturel précis. Dans notre corpus en fait, il existe un ensemble de patronymes qui sont spécifiques à la culture beti mise en texte. Nous pouvons citer entre autres Atangana Mvolo Onguene, Ngo Soha, mère de Kongoligon, Samba Amougou, chef catéchiste de Nlong, Ola, petite sœur de Bella, Souga, Atanagana Ntsama, Biloa, etc. Au final, c'est dire que ce sont des noms de famille dans la culture beti. Ils ont une portée culturelle significative dans la mesure où ils charrient un ensemble de valeurs culturelles spécifiques à ce territoire; ils deviennent donc des « classificateurs de lignée » Lévi-Strauss cité par Mbassi Ateba (2008:34).

9 Cependant, avec l'ouverture du peuple beti à la religion occidentale, on perçoit l'entrée de nouveaux patronymes dans ce milieu, puisqu'après le baptême, le nouveau converti 
reçoit un nom qui est tiré généralement du calendrier chrétien, comme l'explique (Mbengué Nguimé, cité par Maura, 2008) :

L'action anthroponymique des missionnaires s'opérait toujours au moment $\mathrm{du}$ baptême d'un fidèle protestant ou de la prise de la première communion, quand il s'agissait d'un fidèle catholique, la démarche assez simple, consistait à donner un prénom européen, très souvent français, à tout indigène dont la conversion au christianisme devenait effective [...]. Sous l'égide des autorités religieuses, beaucoup d'indigènes subissaient la modification de leurs noms. Les missionnaires travaillaient en étroite collaboration avec le régime colonial dans ce sens, sous prétexte que les noms africains étaient difficiles à prononcer et à retenir (p.84).

Ainsi, parce que ce nom ne relève pas de leur environnement socioculturel immédiat, les individus, par le procédé de " relexicalisation » qui consiste en une mauvaise prononciation, les adaptent selon leur univers culturel. Ce procédé est, selon Mendo Ze (2017), " un malpropisme consistant en une déformation du [nom], due à l'ignorance " (p.127). Dans le corpus justement, les cas sont légion. Nous les récapitulons dans le tableau ci-contre :

Tableau 1

\begin{tabular}{|l|l|}
\hline Prénoms & Prononciation en langue beti ou Betusisation \\
\hline Engelbert & Enguelbette, Enguel (p.5) \\
\hline Richard & Herissar (p.76) \\
\hline Bernard & Banat (p.5) \\
\hline Maman Jeanne & MemaYoana (p.71) \\
\hline Zacharie & Tsakreas (p.71) \\
\hline Thomas & Tomass (p.5) \\
\hline Hubert & Voubette (p.109) \\
\hline Joseph & Yosseb (p.70) \\
\hline Jacob & Yakop (p.5) \\
\hline Maman Rose & Mema Horrosa (p.147) \\
\hline
\end{tabular}

[Les] noms ne sont pas choisis au hasard : chez nous le nom n'est rendu que par sa signification qui le rend agréable, peut-être répugnant aux ancêtres, donc au cosmos, et qui par conséquent lui permet de remplir totalement sa fonction de nommer, c'est-à-dire de mettre au monde [...]. Chaque nom est un sens parce qu'il rythme un sujet dont le destin est comme tracé par ses sonorités et sa musicalité (p.161) ; 
alors, les anthroponymes utilisés ici sont des noms spécifiques au territoire beti. Cette réalité est d'autant plus rendue par leur " consonance " qui a des particularités spécifiques à cette aire culturelle.

12 Au demeurant, ces toponymes et anthroponymes ne relèvent pas d'un fait de hasard. Car, ils fonctionnent, selon Nzessé, comme " des marques ethnostylistiques du texte » (Nzessé, 2012 : 161). Par leur seul usage, ils contribuent à ancrer le roman de Nkoa Atenga dans l'univers socioculturel beti. Étant donné que chaque peuple a ses croyances, ses modes de pensée, de faire et de se comporter, l'ancrage culturel du roman en terroir beti suppose la prise en compte de quelques attributs culturels de cet espace.

\subsection{Quelques attributs culturels beti}

13 Nous entendons par attributs culturels ici, l'ensemble des éléments culturellement marqués qui peuvent rendre compte d'une façon propre de faire, de pensée, d'être, de se vêtir, de se comporter, etc. Dans le roman de Nkoa Atenga, on voit un ensemble d'éléments qui fonctionnent ainsi comme des ethnostylèmes culturels tels que le conçoit Mendo Ze (2017: 109) : "Ils relèvent des considérations relatives à l'art, à l'architecture, et concernant les éléments identitaires et autochtoniques, l'endogénéité, le patrimoine, l'hypoculture occurrente d'où provient l'auteur, et dans laquelle il puise pour former son univers de représentation et donner vie à ses personnages, etc. » $\mathrm{Au}$ nombre de ces éléments, nous avons l'un des instruments de musique traditionnels beti.

\subsubsection{Le tam-tam comme mode d'expression}

14 Comme l'écrit Mendo Ze (2017: 123), le tam-tam « ressortit à la communication comme véhicule des messages à partir d'un instrument de fabrication locale pour l'appel, la danse, la convocation au rassemblement, l'annonce des messages, etc. »Ces différentes fonctions sociales du tam-tam en milieu beti sont fort perceptibles dans notre corpus pour les initiés en matière de décodage des différentes sonorités et des messages qu'ils véhiculent.

En effet, le roman de Nkoa Atenga s'ouvre par la cérémonie funéraire du héros, Sankolo Onana, notable de la tribu Mvog-Ebode. L'annonce du deuil doit être véhiculée par son frère Kongoligon. Voici ce que le narrateur écrit à propos :

Premier des devoirs de responsabilité à cette altitude et dans ces exceptionnelles circonstances : annoncer le deuil à la contrée. Du haut du plus gros, du haut du plus puissant de ses trois tam-tams de notable reconnu, les tam-tams hérités de son patriarche de géniteur, et en digne successeur incontesté de celui-ci, Kongoligon, de ses mains expertes et toujours alertes, l'a fait dans les règles de la tradition ancestrale en la matière. Et non seulement il l'a fait ainsi mais il s'est, de surcroît, l'oreille finement attentive, assuré que cette annonce a bien été répercutée par divers autres tam-tams des villages alentours, relayés eux-mêmes de hameau à hameau dans le même souffle de solidarité (Le Sorcier : 13).

On le voit, le tam-tam est utilisé pour communiquer la triste nouvelle à toute la population de Ngoumlekong. Tous les habitants, initiés aux différentes sonorités de cet instrument de communication locale, affluent vers le lieu du deuil. Le narrateur rend justement compte de cette mobilisation populaire en ces termes : 
Qu'ils arrivent... Qu'ils affluent de toutes parts... Qu'ils arrivent... Qu'ils affluent de partout... De toutes les tribus descendantes d'Ebode, d'Onana, de Mvondo... Qu'ils arrivent... Qu'ils affluent de toutes les tribus auxquelles parviendra cet appel $\mathrm{du}$ tam-tam... Qu'ils arrivent de partout, hommes et femmes... Sankolo Onana... Fils d'Onana Mvondo... De la tribu des Mvog-Ebode est de tout son corps raide allongé... Son corps raide allongé... raide allongé... Sankolo Onana... De la tribu des MvogEbode (Le Sorcier : 12).

17 On constate ainsi que le tam-tam est un langage ; non seulement sa sonorité peut annoncer le deuil dans cette localité, mais aussi, et surtout, il est un appel à la mobilisation du peuple, au ralliement et à l'expression de la solidarité des populations locales, puisque quelques minutes après le retentissement des sonorités, « des hommes accourus [...] de part et d'autre à la salle mortuaire » (Le Sorcier : 13).

Bien plus, l'auteur de Le Sorcier signe et persiste justifie cette prédisposition au décodage, au décryptage des sonorités du tam-tam comme étant la manifestation d'une initiation préalable. Cette coutume est le «Ndan » qu'il explique en note de bas de page du roman en ces termes :

NDAN : « formule métaphorique d'identification en image parfois pittoresque qu'à la naissance, ou peu après, l'enfant généralement le mâle, reçoit de son père et lui permettant, à l'aide du tam-tam, non seulement de lire et de traduire des messages à lui destinés en personne, mais aussi de transmettre et de signer sans équivoque aucune ses propres messages (Le Sorcier : 14 ).

\subsubsection{Légendes et épopées beti}

Selon le dictionnaire Le Grand Robert, l'épopée se définit comme un " long poème (et plus tard, parfois, récit en prose de style élevé) où le merveilleux se mêle au vrai, la légende à l'histoire, et dont le but est de célébrer un héros ou un grand fait ». Dans la société beti, on note la présence d'un personnage épique au nom de Mvondo Ebode Malla, "tout puissant grand-père » de Sankolo. Ce personnage épique lutte courageusement et autoritairement contre toute attitude, toute force, fût-elle anthropomorphe, zoomorphe, surnaturelle, qui veuille déstabiliser son village. Il s'agit concrètement des Français qui tentent de pénétrer le terroir.

Mvondo Ebode Malla, c'est vrai, devenait invisible, face à tout danger mortel inévitable... Mvondo Ebode Malla, nul ne l'ignorait, ne se levait point de son siège de patriarche pour répondre à quelque appel de la nature que ce soit... Mvondo Ebode Malla, oui, expédiait à l'endroit idoine quiconque affrontait son regard ensorceleur en ces moments-là et à ces fins-là... Mvondo Ebode Malla, nul doute, se faisait proprement obéir au doigt, à l'œil et davantage encore au coup de bouc qu'il avait léger, en face des étrangers surtout... (Le Sorcier : 23)

On note aussi la présence de l'épopée de Ndzan'nga Zoa. La légende raconte justement à propos :

Sentant sa mort prochaine, l'un des baobabs recensés de la sagesse dite beti, NDZAN'NGAZOA, chef traditionnel de son état, élabora un véritable et sublime traité complet de relations sociales avant la lettre qu'il entreprit, usant et abusant oralement de toutes les figures de style que lui dictait sa vaste et divergente intelligence, de faire savoir pour application à NOM NGAWONO, celui de ses fils à qui il avait décidé de confier sa succession. Lequel fils n'avait qu'à répondre non ou oui à des interrogations qui résumaient parfaitement la philosophie de l'acte à poser selon les cas de figure et qui entraînaient tout naturellement, comme cela allant de soi, ces réponses que le père avait pourtant et soigneusement préparées à l'avance. Authentique code de conduite et de gestions des affaires tant domestiques, 
familiales, claniques et tribales que de subordination au Blanc, ce chef-d'œuvre de la littérature orale que célèbrent de nos jours encore de grands virtuoses du muet, à l'instar de EBOLO NOAH, est passé à la postérité sous la dénomination d'Épopée de NDZAN'NGAZOA... (Le Sorcier : 106)

21 À côté de ces épopées, on a la légende de la colline des chimpanzés. Elle remonte entre la fin du XVIIIe siècle et le début du XIXe siècle, et raconte une bagarre spectaculaire entre deux groupes de gorilles et de chimpanzés, au sujet de la défense et de l'invasion du territoire :

Les collines des chimpanzés. Qui tenaient leur nom et davantage encore leur renommée de la légende locale. La légende locale qui soutient depuis toujours que ces sommets pelés comme une pierre qui roule et qu'aucun œil, prévenu ou non, ne peut manquer d'embrasser de jour à partir de la petite cité campagnarde d'Okola, doivent ce singulier spectacle à la suite d'une bagarre tout aussi légendaire. Une bagarre entre deux " armées » de gorilles et de chimpanzés désireux, dans un camp comme dans l'autre, de s'assurer en exclusivité la suprématie des lieux alors paradisiaques, et dont on situe l'occurrence pendant le mouvement migratoire des Eton dans la région (Le Sorcier : 193).

Au final, ces légendes et épopées constituent et irradient l'imaginaire de membres de la communauté. Elles sont restituées ou du moins célébrées dans des cantiques, des berceuses, chants populaires, etc. Ceci permettant d'entretenir la pensée de tous et d'orienter, de guider et de réguler le comportement des uns et des autres.

\subsubsection{L'insertion de la langue eton}

Dans cette partie, nous nous intéressons au phénomène linguistique comme élément d'ancrage culturel. Bien qu'écrivant en français, Camille Nkoa Atenga utilise plusieurs expressions étrangères qui permettent de situer son roman dans son contexte d'énonciation. Il s'agit en fait des emprunts, définis comme des " éléments qui passent d'une langue à une autre, s'intègrent à la structure lexicale, phonétique et grammaticale de la nouvelle langue et se fixent dans un emploi généralisé de l'ensemble des usagers que ceux-ci soient bilingues ou non » (Ngalasso, 2001 cité par Nzessé, 2012 : 169). Nous observons, entre autres, l'intrusion des termes suivants : Ndan (p. 14)/Sonorité du tam-tam servant d'identification d'un enfant ; Ntangani (p. 17)/Le Blanc; Obom (p. 19)/Tissu d'habillement obtenu à partir de l'écorce d'un jeune arbre ; Bila (p. 20)/Cache-sexe ; Abouga (p. 21)/Pipe artisanale ; Sa (p.25)/Prune ; Ngega (p. 33)/Fruit sauvage ; Helmett (p. 39)/Casque colonial ; Bissep (p. 42)/Années ; Bekon (p. 44)/Les fantômes et les séjours des morts ; Mindil-Ebul (p. 54)/Neuf toitures ; Oyon (p. 62)/Les quatre saisons climatiques du mois d'août ; Akogo Ebena (p. 88)/Désigne des vieilles filles ; Zezeamininga (p. 90)/Une femme réduite en rien ; Mbog (p.89)/Écureuil ; Essamseguele (p. 92)/Vulgaire, vulgarisé, vulgivaque ; Biyom bi mô (p. 98)/Des biens palpables, visibles et concrets ; Bikut-si (p.115)/Danse populaire ; Nga tara (p. 145)/ Épouse de papa ; A bot bese (p. 145)/À tous les humains ; Ekutésiki (p. 153)/Il n'y a rien à dire ; Ngomna (p.158)/Administrateur, l'autorité supérieure du commandement territorial ; Nala (p. 166)/Voilà... Bravo ; Komé (p. 270)/Noisette ; A bot bambese/Cri d'exclamation, d'étonnement de saisissement au superlatif qui signifie " ô tous les miens "; Ndomba (p.211)/Perle de l'art culinaire. C'est un plat fait à base essentiellement de viande de chèvre et de silure conditionné dans des feuilles de bananiers ; Mboni (p.204)/Noix de coco ; Ekoko owono (p. 196)/Pâte d'arachide. 
$24 \mathrm{Au}$ demeurant, tous ces termes, idiomes empruntés à la langue eton concourent à ancrer le roman de Nkoa Atenga dans ce milieu socioculturel. Il se dégage une écriture polyglotte (français et eton) dans laquelle « les barrières du français s'ouvrent [à un autre code], faisant du texte un faisceau linguistique objectif à large spectre parce que comportant des tournures consacrées et (re) connues par le lecteur » (Ngetcham, 2011 : 94). Cette langue influe les habitudes linguistiques de l'écrivain camerounais dans sa créativité et impose le décodage par le lecteur de ces unités lexicales de telle sorte que le choix de ces lexies, "directement puisées dans [cette langue nationale], est déterminé par la culture [de l'écrivain] et a pour fonction de "faire couleur locale » (Ullmann, 1975: 163) ou encore de "plonger le lecteur immédiatement dans une atmosphère culturelle particulière » (Ngalasso, 1984 : 18-

19) »: l'univers beti précisément.

\section{Les parémies beti}

Notre tâche dans cette articulation de notre réflexion est de mener une étude structurale des parémiologies en contexte beti. Avant de le faire, un relevé exhaustif de ces parémies semble nécessaire.

\subsection{Définition et recensement}

Les parémies ou énoncés sentencieux (proverbes, dictons, adages, maximes, etc.) sont des formules figées qui renferment la sagesse populaire sous forme de phrases laconiques et faciles à mémoriser qui sont passées de génération en génération par le truchement de la tradition orale. Selon Maloux (1960: 110), « le proverbe désigne une vérité morale ou de fait, exprimé en peu de mots, ou bien une expression imagée de la philosophie pratique, ou bien une parole mémorable, ou encore un vers ou un distique célèbre passé en proverbe ». Kabuta apporte un supplément de sens lorsqu'il précise le consensus du savoir partagé et validé par la communauté. Il écrit justement à propos :

Le proverbe est une formule qui exprime une vérité d'expérience. Il est un résumé, un raccourci de toute une réflexion. Il y a consensus de la communauté - du moins des membres les plus instruits sur son contenu, qui ne peut être consciemment remis en question. Il dispense de faire de longs développements sur un grand nombre de situations courantes. (Kabuta, $1997: 69$ )

27 Ainsi, le consensus social fait que chaque langue véhicule une cohorte de pensées comme une sorte de mémoire embarquée, de tradition intériorisée. Les proverbes donnent ainsi quelques éléments sur le conscient collectif d'une communauté, sa mentalité, ses habitudes, ses besoins, son contexte géographique. Considérés comme des " dits de vieux » (Oihénart, 1992: 129), " leur caractéristique essentielle réside dans l'anonymat de leur auteur, même si l'on peut circonscrire l'environnement dans lequel ils sont conçus ou évaluer l'adéquation de leur utilisation à son contexte " (Ngetcham, 2010 : 94). C'est donc dire que les parémies ne sont utilisées que dans des sphères socioculturelles spécifiques et nécessitent la prise en compte des référents endogènes dans leur processus de signification.

28 À la lecture de Le Sorcier signe et persiste de Camille Nkoa Atenga, on est marqué par la présence fulgurante des proverbes beti. L'auteur, mué en patriarche, use de ce genre de la tradition orale pour entretenir les valeurs partagées dans (et par les membres de) la 
communauté beti. Dans l'œuvre justement, on en note vingt-trois précisément. Nous les synthétisons dans le tableau suivant à deux entrées ; d'une part l'extrait introducteur (qui peut venir avant le proverbe et parfois après) et, d'autre part, le proverbe proprement dit.

Tableau 2

\begin{tabular}{|c|c|}
\hline Extraits introducteurs & Proverbes \\
\hline $\begin{array}{l}\text { Qui prétendait l'ignorer ici où l'imaginaire } \\
\text { populaire soutient depuis toujours que }\end{array}$ & $\begin{array}{l}\text { l'on ne pleure réellement que ses morts à l'occasion } \\
\text { des deuils (p. 8) }\end{array}$ \\
\hline $\begin{array}{l}\text { Cette solidarité que la sagesse populaire a } \\
\text { immortalisée et célèbre ici par ce dicton } \\
\text { plus que topique qui clame qu' }\end{array}$ & $\begin{array}{l}\text { une seule main ne peut permettre de grimper à qui } \\
\text { que ce soit (pp. 12-13) }\end{array}$ \\
\hline RAS & $\begin{array}{l}\text { Brûlé une fois, honte à lui ; brûlé deux fois, honte à } \\
\text { nous (p. 17) }\end{array}$ \\
\hline $\begin{array}{l}\text { Ne soutient-on pas ici depuis des lustres et } \\
\text { par un joyeux euphémisme que }\end{array}$ & $\begin{array}{l}\text { le neveu trouve jouissance auprès de l'épouse de son } \\
\text { oncle (p. 18) }\end{array}$ \\
\hline Avance, précède-moi... & $\begin{array}{l}\text { Il n'y a jamais de sécurité assurée pour l'éléphanteau } \\
\text { qui suit l'éléphant (p. 32) }\end{array}$ \\
\hline $\begin{array}{l}\text { Ce que la sagesse beti formule dans le } \\
\text { célèbre et célébré dicton populaire qui dit }\end{array}$ & $\begin{array}{l}\text { Rien de plus normal, rien de plus conseillé pour } \\
\text { l'individu que de répandre l'humus autour de son } \\
\text { pied de macabo avant tout autre (p. } 36 \text { ) }\end{array}$ \\
\hline $\begin{array}{l}\text { Adam Smith aurait-il été Beti sans le savoir, } \\
\text { dites-vous ? Lui qui a toujours soutenu, sans } \\
\text { être vraiment démenti jusqu'à ce jour, que }\end{array}$ & $\begin{array}{l}\text { c'est en poursuivant son propre intérêt que } \\
\text { l'individu favorise souvent l'intérêt de la société } \\
\text { (p. 36) }\end{array}$ \\
\hline Bessiga... avait retenu de son père que & $\begin{array}{l}\text { quand un enfant - le fils aîné en l'occurrence - est } \\
\text { plus malheureux que son père, c'est la détresse et la } \\
\text { désolation au point le plus insoutenable et haïssable } \\
\text { (p.36) }\end{array}$ \\
\hline $\begin{array}{l}\text { soutient depuis des lustres un proverbe beti } \\
\text { bien populaire }\end{array}$ & Le cœur ne vieillit jamais (p. 55) \\
\hline $\begin{array}{l}\text { Accroché solidement qu'il demeure à ce } \\
\text { credo largement partagé par la gent } \\
\text { masculine et soutenant qu' }\end{array}$ & $\begin{array}{l}\text { on ne cesse pas d'aimer sa femme parce qu'on la } \\
\text { trompe ou plutôt parce qu'on accepte les faveurs } \\
\text { sollicitées d'une autre femme (p. } 55 \text { ) }\end{array}$ \\
\hline RAS & $\begin{array}{l}\text {... le meilleur moyen de faire front à tous [1] est défis } \\
\text { naturels... c'est [...] d'éviter l'ignorance (p. 63) }\end{array}$ \\
\hline $\begin{array}{l}\text { Mais dois-je déduire de ta présence ici que } \\
\text { tu serais déjà persuadée que }\end{array}$ & $\begin{array}{l}\text { le meilleur bain est celui du soir, après le travail et } \\
\text { avant le repas (p. 65) }\end{array}$ \\
\hline $\begin{array}{l}\text { avait cru devoir rappeler Mema Sessana à sa } \\
\text { fille Bella }\end{array}$ & $\begin{array}{l}\text { Les grands regards, surtout quand ils sont } \\
\text { nombreux, dissuadent toujours quand ils } \\
\text { n'annihilent pas purement et simplement toute } \\
\text { velléité d'action maléfique (p. 95) }\end{array}$ \\
\hline
\end{tabular}




\begin{tabular}{|c|c|}
\hline $\begin{array}{l}\text { Ce que, dans toute la région, traduit avec } \\
\text { éloquence ce célèbre proverbe popularisé } \\
\text { jusque dans le "Bikut-si » et proclamant } \\
\text { sans détour qu' }\end{array}$ & $\begin{array}{l}\text { en toute chose, priorité doit être accordée à la } \\
\text { famille proche et directe (p.115) }\end{array}$ \\
\hline $\begin{array}{l}\text { En respectant souvent le vieil adage de « } \\
\text { Qui se ressemble s'assemble " que le Beti } \\
\text { traduit, lui, ici, par }\end{array}$ & $\begin{array}{l}\text { Les troupeaux de buffles à part et les troupeaux } \\
\text { d'éléphants à l'écart... (p. 141). Traduction en bas de } \\
\text { page, en beti : NYAT, NYAT, MENGOS... ZOK, ZOK, } \\
\text { MENGOS }\end{array}$ \\
\hline Attention cependant... Kongoligon & $\begin{array}{l}\text { N'est pas du tout l'huile de palme à réchauffer au } \\
\text { préalable (p. 142) }\end{array}$ \\
\hline Est-il possible & $\begin{array}{l}\text { De donner un coup de pied violent à la fourmilière } \\
\text { sans que les fourmis s'en échappent et éclatent dans } \\
\text { toutes les directions? (p. 145) }\end{array}$ \\
\hline RAS & $\begin{array}{l}\text { Les gros poissons ne se mangent pas entre eux } \\
\text { (p. 184) }\end{array}$ \\
\hline $\begin{array}{l}\text { Car, disait-on souvent dans ces } \\
\text { circonstances }\end{array}$ & $\begin{array}{l}\text { en visant le rat - surtout avec une pierre à écraser- } \\
\text { on peut facilement casser de précieuses jarres d'eau } \\
\text { alignées contre le mur (p. 185) }\end{array}$ \\
\hline [Sankolo]... tenait ceci de son père qu' & $\begin{array}{l}\text { il ne faut jamais soulever la natte chez quelqu'un, } \\
\text { aîné de surcroît, pour montrer la poussière qui s'y } \\
\text { trouve (p. 196) }\end{array}$ \\
\hline RAS & $\begin{array}{l}\text { Qui se frotte à l'épine doit s'habituer à la vue de son } \\
\text { sang (p. 197) }\end{array}$ \\
\hline RAS & $\begin{array}{l}\text { Ne pas fuir l'éléphant au seul écho du bruit de son } \\
\text { approche (p. 217) }\end{array}$ \\
\hline RAS & $\begin{array}{l}\text { Il est quelquefois indiqué d'oublier ce que l'on sait... } \\
\text { (p. 275) }\end{array}$ \\
\hline
\end{tabular}

$\mathrm{Au}$ demeurant, force est de constater que Nkoa Atenga se mue en un patriarche, dépositaire des savoirs et des sagesses. Il entend les partager avec les membres de sa communauté. Son texte se présente alors comme l'« expression de l'idéologicoculturel »

(Fosso, 2004 : 49) qui « fournit des renseignements précis sur les mœurs, les modes de pensée et de vie du groupe social » (Nzessé, 2012: 248). Ce qui impose, pour un décryptage sémantique complet, une analyse au niveau stylistique.

\subsection{Analyse structurale}

Dans le cadre de cette analyse structurale des proverbes, nous allons nous intéresser à deux éléments à savoir les temps et modes verbaux et les sujets et les prédicats. S'il est bien entendu que les proverbes se caractérisent par l'anonymat de leur auteur, cela suppose que la sagesse transmise est partagée par les membres de la communauté. Ce qui justifie l'emploi régulier du présent comme temps et de l'indicatif comme mode. Dans les vingt-trois proverbes recensés dans notre corpus, le présent de l'indicatif revient sensiblement dans toutes les occurrences. Il s'agit en fait d'une forme verbale 
dépourvue de marque temporelle et qui n'a d'autres significations que ce qui va de soi et donc immédiat. Il s'agit du temps que le locuteur, nous voulons dire le membre du groupe social dans lequel le proverbe est tenu, voit comme sien, le temps présent ; d'où son implication dans le décodage et l'intégration des valeurs et des sagesses transmises. Cette neutralité prédispose le proverbe à l'expression de vérités générales. On a donc affaire à un présent de vérité générale, omni-temporel ou encore le présent gnomique. Le terme gnomique vient justement du grec gnômikos qui signifie "sentencieux ». De manière logique, il concerne les sentences, les maximes, les axiomes, les proverbes, les adages, et les autres lois universelles. Il paraît donc justifier que les proverbes de notre corpus soient conjugués fréquemment au présent de l'indicatif.

Le second poste d'analyse concerne le sujet dans les différents proverbes. Il s'agit du terme considéré comme le point de départ de l'énoncé. Parce que formules figées rendant compte d'une sagesse populaire, les proverbes s'énoncent régulièrement à un mode impersonnel quand ils n'expriment pas des singularités à valeur généralisante. Il s'agit tout simplement des procédés stylistiques par lesquels chaque membre de la communauté puisse s'identifier aux conseils, aux vérités et aux expériences portés par eux. Ces valeurs pouvant être la solidarité ("Une seule main ne peut permettre de grimper à qui que ce soit »), la responsabilité des parents dans l'éducation des parents (" Il n'y a jamais de sécurité assurée pour l'éléphanteau qui suit l'éléphant "), l'assistance mutuelle de soi (Les gros poissons ne se mangent pas entre eux), assomption des actes posés (« Qui se frotte à l'épine doit s'habituer à la vue de son sang "), etc.

$\mathrm{Au}$ final, ces aphorismes, intériorisés par les membres du groupe, régulent le comportement de ces derniers et concourent à l'expression d'une identité locale.

\section{Parémies et expression identitaire beti}

Il est question à ce niveau de notre analyse, de montrer l'impact ou du moins l'incidence des savoirs véhiculés, des conseils et d'expériences transmis par les parémies dans la construction des identités des personnages. Et par-delà, nous nous intéresserons au rôle de l'écrivain-conteur africain.

\subsection{Les proverbes comme régulateurs des comportements sociaux}

L'usage des parémies ne relève pas du hasard, de l'art de la figuration. En effet, les parémies, utilisées dans son contexte d'énonciation, donnent une valeur rhétorique supplémentaire puisqu'elles fonctionnent comme des arguments d'autorité. Ainsi, chaque séquence narrative du récit à caractère argumentatif s'adosse sur le proverbe. C'est qu'affirme d'ailleurs Jean-Christophe Kasende :

Il est une assertion-preuve toujours disponible pour servir de caution à une pratique sociale précise. Bref, en tant qu'intertexte et idéologème, chacun des proverbes entretient avec l'argumentation de la séquence narrative à laquelle il se rapporte une relation d'affirmation ou de négation. En d'autres termes, chaque énoncé de l'oralité traditionnelle impliquée dans le jeu intertextuel est un argument à l'appui d'une idéologie contre une autre idéologie. Par induction narrative, les proverbes apparaissent dans leurs contextes comme « ces passages où, plus au moins explicitement, l'auteur, le narrateur, le poète énonce des idées tout en prenant position » (Dubois, 1994 : 235). C'est par cette stratégie énonciative 
intertextuelle en effet que l'énonciateur détermine le système axiologique des instances actantielles et suggère métalinguistiquement un modèle qualificatif qui lève l'ambiguïté $d u$ récit. Le proverbe aide donc à supposer, à partir des règles $d u$ système sur lequel il se fonde, qu'une attitude sociale décrite dans le récit est classée parmi les actes positifs ou négatifs, permis ou interdits. (2007 : en ligne)

Dans notre corpus justement, le proverbe est modulable en fonction de sa circonstance d'énonciation. Face à la triste nouvelle de la mort d'Onana Sankolo, ses épouses se lamentent. Pratique habituelle puisque dans la collectivité, les veuves dans le cas précis savent que " l'on ne pleure réellement que ses morts à l'occasion des deuils " (Le Sorcier, 8). Dans ces instants pénibles, les attristées sont soutenues par les autres membres de la communauté. Ce qui appelle donc à la solidarité, valeur ancestrale. Celle-ci est rendue dans la sagesse populaire suivante : « Rien de plus normal, rien de plus conseillé pour l'individu que de répandre l'humus autour de son pied de macabo avant tout autre » (Le Sorcier, 36). Depuis le mariage de Bella avec Sankolo, celle-ci est exposée aux combats qui ne sont pas toujours à son niveau (des combats physiques et parfois mystiques). Dans cette situation, sa maman, en vieille femme beti avisée, lui dit: "Les grands regards, surtout quand ils sont nombreux, dissuadent toujours quand ils n'annihilent pas purement et simplement toute velléité d'action maléfique (Le Sorcier, 95).

L'exemple le plus intéressant vient de l'attitude de Mema Yoana. Lorsque Sankolo est arrêté par l'administration coloniale pour insoumission à l'autorité religieuse, les colons veulent lui retirer le » collier sacré », fait en dent de panthère qu'il a hérité de son père ; celui-ci l'ayant prédestiné à défendre les valeurs cardinales de la tribu beti. Le catéchiste Enguelbette demande la position de Mema Yoana, une conseillère très écoutée pendant les tournées pastorales. Elle est confrontée, elle, en tant que femme beti, descendante de ces ancêtres, à un dilemme : doit-elle dire la vérité à l'autorité religieuse ou pas ? Pendant ses interminables questionnements, elle se rappelle de cette vieille sagesse populaire que lui recommandait de retenir par-dessus tout sa mère, qui stipulait que : «Il est quelquefois indiqué d'oublier ce que l'on sait... » (Le Sorcier, 275). Irradiée par cette leçon ancestrale, et "nonobstant l'affection tout à fait maternante qu'elle éprouvait pour son curé, malgré l'attachement jamais pris en défaut ", elle n'a pas trahi la valeur ancestrale. Au final, elle est convaincue de ce que l'on ne peut se soustraire aux valeurs sacrées de sa tradition. Même en situation de contact avec l'altérité occidentale - Française notamment qui ici avait des projets d'assimilation des indigènes -, on ne peut ignorer le culte voué aux ancêtres et à leur sagesse parce que transmise à la postérité dont elle fait partie: "À la femme beti qu'elle était, la sagesse des ancêtres, relayée sans ride de génération en génération, avait sans cesse susurré et seriné que non seulement on ne se dépouille que de ce que l'on remplace, mais aussi, et surtout que l'on ne se dépouille pas de ce qui appartient à la communauté » (Le Sorcier, 273).

En somme, on note que le comportement des personnages dans le récit est guidé par la sagesse véhiculée par le proverbe. Ce dernier fonctionne alors comme un discours "préconstruit» (Pêcheux, 1975 : 114). Posé comme une évidence doxale, le proverbe acquiert toute sa force argumentative. C'est dire, en définitive que "l'utilisation de ces aphorismes (dictons, proverbes, adages) relève de l'art de la parabolisation du discours, imposant un décryptage par quiconque désireux de comprendre des énoncés indéchiffrables au premier degré. Un code initiatique non écrit, mais connu dans la collectivité du locuteur et ses interlocuteurs, régit le codage/décodage de ces formules» (Ngetcham, $2011: 102)$. 


\subsection{Le Sorcier signe et persiste : un conservatoire des valeurs beti}

Dans son article « Oralité et narrativité dans le roman africain » (2007, en ligne), JeanChristophe Kasende constate que :

La plupart des univers de romans africains subsahariens sont bâtis sur l'imbrication des genres. Chansons, contes, proverbes et devinettes provenant tout droit de la littérature orale traditionnelle sont convoqués par l'écrivain pour construire l'architecture du roman. Incorporés dans la structure narrative de ce dernier, ces différents genres de l'art oral traditionnel négro-africain fonctionnent comme des références au discours de l'« Autre » par rapport au récit romanesque.

Par-là, on note la survivance de l'oralité en tant qu'expression de la culture traditionnelle africaine. Notons que ce choix scripturaire et esthétique n'est point un fait de hasard dans la littérature africaine. Le romancier, se posant comme patriarche, se sent investi d'une mission: celle de défendre et de pérenniser la tradition orale. Selon Gérard Noumsi (2002 cité par Nzessé, 2012: 10), il s'agit "d'une réponse au problème de communication socioculturelle et au besoin qu'éprouvent les [écrivains] Négro-Africains de transmettre et de pérenniser leur culture et sagesse ancestrales ».

Camille Nkoa Atenga ne s'écarte pas de cette mission. Se considérant comme le patriarche de la communauté beti, le fils du terroir entend, par la textualisation des parémies sues-convoquées et d'autres artefacts de la littérature orale (chants, cantiques, légendes, épopées) et des spécificités culturelles beti (tam-tam, Mvet, us et coutumes, toponymes, anthroponymes, etc.) pérenniser la culture ancestrale. En effet, l'auteur entend " écrire et perpétuer oralement à travers les temps l'histoire dans les moments les plus significatifs du déroulement de la vie des sociétés, des tribus, des clans et même des familles " (Le Sorcier, 10). Son récit devient alors la matérialisation de cet acte de défense des valeurs orales. Par ce procédé, on assiste à « une écriture dite ou, si l'on préfère, une parole codifiée par écrit [de telle sorte que] se pose la question de la primauté de l'auteur disant sur le conteur écrivain » (Ngetcham, 2011 : 102).

$\mathrm{Au}$ final, par des intrusions multiformes qui renseignent sur le contexte d'énonciation, le terroir d'appartenance de Camille Nkoa Atenga, Le Sorcier Signe et persiste devient le lieu de fixation des valeurs culturelles, les traditions, bref l'histoire des Beti. C'est donc un roman qui entend conserver

toutes choses et bien d'autres encore qu'on racontait, vivait et répétait lors des rencontres multiformes à tous les niveaux des générations et qui, sans ces exercices pratiques au cours desquels on élevait au rang de héros les meilleurs chasseurs et autres lutteurs, ne feraient des valeurs culturelles beti que de l'eau stagnante. Sans courant porteur pour l'avenir... » (Le Sorcier, 64).

41 La présente réflexion intitulée « Le discours parémiologique beti dans Le Sorcier signe et persiste de Camille Nkoa Atenga " avait pour but, dans une perspective ethnostylistique, de révéler, à travers l'écriture de l'auteur, le terroir auquel il appartient. L'analyse a tout d'abord consisté, comme le prescrit Mendo Ze, à cerner l'espace d'énonciation du texte littéraire. Nous avons donc montré, à partir d'une étude des toponymes et anthroponymes d'une part, et, d'autre part, les éléments culturels y afférent, que le lieu-source d'énonciation est le terroir beti. Par la suite, l'analyse s'est intéressée à une étude des proverbes beti. La tâche a consisté d'abord à faire un recensement desdits proverbes avant de mener une étude structurale. Sagesse partagée dans (et par les membres de) la communauté, il restait donc pertinent de voir comment ces aphorismes concourent à l'expression de l'identité locale. Nous avons démontré que 
les parémies sont un régulateur des habitudes et comportements sociaux car, elles irradient, guident, les attitudes des personnages dans leur vécu quotidien. Tout compte fait, on déduit que l'écriture de Nkoa Atenga milite pour une certaine pérennisation de la culture beti en particulier et Camerounaise en général en mal d'expression dans un monde occidentalisé. D'où la modification même des perspectives d'analyse du texte littéraire africain, en mettant au premier plan, des approches qui permettront de faire " ressortir la continuité relative du discours traditionnel oral au discours écrit et à examiner la survie de la tradition dans un contexte de modernisation » (Omgba, 2015 : 19).

\section{BIBLIOGRAPHIE}

KASENDE, J-C., " Oralité et narrativité dans le roman africain ", in Éthiopiques n 79, 2007, disponible sur [http://ethiopiques.refer.sn/spip.php?article1563], (page consultée le 28 juin 2017).

MAURA, D., « Les patronymes dans l'histoire des Mafa du Nord-Cameroun : essai d'analyse sociohistorique ", in Littérature orale africaine. Décryptage, reconstruction, canonisation, dans Dili Palaï Clément et Cyr Pangop Alain Kameni (dir (s).), Paris, L'Harmattan, 2013, pp. 73-86.

MENDO ZE, G., Cahier d'un retour au pays natal d'Aimé Césaire : Analyse ethnostylistique, Paris, L'Harmattan, 2010.

MENDO ZE, G., Ethnostylistique. Une analyse néo-structurale, Presses Universitaires d'Afrique, 2017.

NGETCHAM, « Écriture métisse et construction identitaire dans L'A-Fric de Jacques Fame Ndongo ", in Nka', 2011, pp. 89-104.

NKOA ATENGA, C., Le Sorcier signe et persiste, Éditions Sherpa, 2003.

NZESSÉ, L., « Oralité et appropriation du français dans la littérature africaine : le cas de Allah n'est pas obligé d'Ahmadou Kourouma et de La Joie de vivre de Patrice Nganang ", in Synergies $\mathrm{n}^{\circ} 10$, 2010, pp. 245-258.

NZESSÉ, L., " La prégnance du réel dans la fiction contemporaine de l'espace francophone : une lecture ethnostylistique de Moi Taximan de Gabriel Kuiche Fonkou ", in Analyse : Langues, Textes et Sociétés $\mathrm{n}^{\circ} 15,2012$, pp. 159-172.

OMGBA, R-L., " Pour une critique oragraphique ", in Le Texte littéraire africain et sa critique, une publication de la revue internationale de langue et littérature, Écritures, $n^{\circ}$ XII, Yaoundé, CLÉ, 2015, pp. 13-26.

WESTPHAL, B., (2005), « Pour une approche géocritique des textes », in SFLGC (Vox Poetica), disponible sur [http://www.vox-poetica.org/sflgc/biblio/gcr.htm] (page consultée le 18 septembre 2016). 


\section{NOTES}

1. Dans la suite de cette réflexion, nous l'abrégerons Le Sorcier accompagné de la page citée.

2. Dans son article " Pour une approche géocritique des textes ", in SFLGC (Vox Poetica), http:// www.vox-poetica.org/sflgc/biblio/gcr.htm, page consultée le 25 novembre 2015, Bertrand Westphal, montre que la ville textualisée entretient trois types de relations avec le réel : une " relation de transposition signalée par ce qu'on pourrait appeler un "contrat toponymique » (Paris) »; " une relation de transfiguration, revendiquée ou non (Verrières/Besançon) »; « une relation niant tout référent, toute souche toponymique

3. C'est nous qui soulignons ces mots et expressions pour en exergue les indices de référenciation géographique.

4. Dans le roman, Camille NkoaAtenga utilise ce néologisme pour désigner justement ce « malproprisme » qu'on observe dans la prononciation de certains noms chez les Beti.

\section{RÉSUMÉS}

Depuis quelques décennies, on assiste à un renouvellement esthétique dans la littérature africaine francophone en général et camerounaise en particulier. En effet, le contact avec l'altérité occidentale a influencé les modes de faire, de se vêtir, de penser et d'être des peuples africains au point où l'on assiste à la perte des valeurs africaines. Face à ce problème de déclin de la civilisation orale, l'écrivain africain resté au pays, notamment, se mue en patriarche, courroie de transmission et de sauvegarde des valeurs ancestrales. Prenant appui sur Le Sorcier signe et persiste (2003) de Camille Nkoa Atenga, le but ici est de montrer comment l'auteur s'enracine fortement dans l'univers socioculturel beti et se mue en patriarche pour pérenniser la culture beti en particulier et camerounaise en général en mal d'expression dans un monde occidentalisé.

In the last few decades, there has been a renewal of aesthetics in the French African literature in general and Cameroon in particular. Indeed, the contact with the otherness of the west has influenced the ways of doing, dressing, thinking, and being African people to the point where we are witnesing the loss of African values. Faced with this problem of declining oral civilization, the African writer remained in the country in particular, becomes a patriarch, belt transmission and safeguarding ancestral values. Based on Camille Nkoa Atenga's Le Sorcier signe et persiste (2003), the aim here is to deeply rooted in the beti socioculural universe and is transformed into a patriarch to perpetuate beti culture in particular and cameroon in general in poor expression in a westernized world.

\section{INDEX}

Mots-clés : parémie, terroir, expression culturelle, transmission

Keywords : paremia, terroir, cultural expression, transmission 
AUTEUR

MOUHAMADOU NGAPOUT KPOUMIÉ

Université de Dschang, Cameroun 